Article

\title{
Self-Assembly of Pyridine-Modified Lipoic Acid Derivatives on Gold and Their Interaction with Thyroxine (T4)
}

\author{
Willem M. Albers ${ }^{1, *}$, Roberto Milani ${ }^{1,2,3, *}$, Kirsi Tappura ${ }^{1}$, Tony Munter ${ }^{1}$, \\ Giuseppe Resnati $^{2,3}$ and Pierangelo Metrangolo ${ }^{1,2,3}$
}

1 VTT Technical Research Centre of Finland, Sinitaival 6, 33720 Tampere,

Finland \& Metallimiehenkuja 8, Espoo, 02044 VTT, Finland; E-Mails: kirsi.tappura@vtt.fi (K.T.); tony.munter@vtt.fi (T.M.); pierangelo.metrangolo@polimi.it (P.M.)

2 NFMLab-DCMIC “Giulio Natta”, Politecnico di Milano, Via Mancinelli 7, I-20131 Milano, Italy; E-Mail: giuseppe.resnati@polimi.it

3 Center for Nano Science and Technology@PoliMi, Istituto Italiano di Tecnologia, Via Giovanni Pascoli, 70/3, I-20133 Milano, Italy

* Authors to whom correspondence should be addressed; E-Mails: martin.albers@vtt.fi (W.M.A.); roberto.milani@vtt.fi (R.M.); Tel.: +358-20-722-3318 (W.M.A.); +358-20-722-7175 (R.M.).

Received: 26 December 2012; in revised form: 30 January 2013 / Accepted: 31 January 2013 / Published: 6 February 2013

\begin{abstract}
Pyridyl derivatives of lipoic acid were prepared as ligands for the study of the interaction with thyroxine (T4). Thin self-assembled films of the ligands were prepared in $70 \%$ ethanol on gold and their interaction with $\mathbf{T} 4$ was studied by titration experiments in an aqueous buffer solution using Surface Plasmon Resonance (SPR). The thickness and refractive index of the ligand layers were calculated from SPR spectra recorded in two media, also allowing for surface coverage and the density of the layers to be estimated. Two ligands, a 4-pyridyl and a bis(2-hydroxyethyl) derivative of lipoic acid, were selected to investigate the feasibility for producing molecularly imprinted self-assembled layers on gold for T4. The methodology was to co-assemble T4 and the ligand onto the gold surface, elute the $\mathbf{T} 4$ from the layer under alkaline conditions, and study the rebinding of $\mathbf{T} 4$ to the layer. Multiple elution/rebinding cycles were conducted in different buffer solutions, and rebinding of T4 could be observed, with a moderate binding affinity that depended greatly on the solvent used. More optimal binding was observed in HBS buffer, and the affinity of the interaction could be slightly increased when the 4-pyridyl and bis(2-hydroxy-ethyl) derivatives of lipoic acid were combined in the imprinted layer.
\end{abstract}


Keywords: thyroxine; self-assembly; imprinting; noncovalent interactions; Surface Plasmon Resonance (SPR)

\section{Introduction}

Thyroxine (or 3,5,3',5'-tetraiodo-L-thyronine, T4) and triiodothyronine (or 3,5,3'-triiodo-L-thyronine, T3) (Figure 1) are the two major thyroid hormones related to thyroid action, which is essential for the differentiation and growth of nearly all tissues, while also having marked influence on oxygen consumption, metabolic rate and RNA synthesis [1]. Their synthesis, secretion and transport are coordinated by the hypothalamus, pituitary and thyroid glands, and comprise various receptors, transport proteins and additional hormones. Thyrotropin Releasing Hormone (TRH) and Thyroid Stimulating Hormone (TSH) are the main regulators of the biosynthesis of T4 and T3 by a negative feedback mechanism. T4 is synthesized in the thyroid gland by iodination and further covalent attachment of the phenyl rings from the amino acid tyrosine, while $\mathbf{T 3}$, the more potent hormone, is produced from $\mathbf{T} 4$ by deiodination outside the thyroid gland [2].

Thyroid hormones are known to bind to various receptors, of which many crystallographic structures have already been elucidated [1]. Since these compounds are very hydrophobic, they are generally bound with high affinity into a hydrophobic pocket of the thyroid receptors $[3,4]$, and their concentrations in blood serum are notoriously low $(1.5-20 \mathrm{pM})$ [5]. Various carrier proteins are capable of solubilizing hydrophobic compounds, including a large collection of common drugs and vitamins like retinol. The main transporters of thyroid hormones in the blood stream are Thyroxine Binding Globulin (TBG), albumin, and transthyretin (pre-albumin) [3,6,7]. In particular, the binding of T4 to transthyretin seems to involve not only hydrogen bonds, but also short I $\cdots$ O contacts [8], which can be identified as genuine halogen bonds, namely, noncovalent interactions involving halogen atoms as electrophilic species [9].

The attention to the biological relevance of T4-analogue halocarbons is strongly growing. It has been demonstrated that poly-brominated diphenyl ethers (PBDEs) show a remarkable thyroid hormonal activity as endocrine disrupting compounds (EDCs) [10]. This is not surprising when taking into account the structural similarities of PBDEs with T4 (see Figure 1); however, the exact mechanism of interference with the thyroid hormonal action is not yet fully understood. The detection of T4 and its analogues is therefore an important societal issue. Nowadays, in clinical practice, free T4 and its mono-dehalogenated analogue $\mathbf{T 3}$ are measured by various highly sensitive methods, such as radioimmunoassay (RIA), fluoroimmunoassay (FIA and TR-FIA) [11] or chemiluminescent assay. Recently, EDCs have been assayed with biosensor systems, e.g., utilizing thyroid transport proteins as receptors for EDCs [12,13]. 
Figure 1. Molecular structures of T4 (left), T3 (mid), and the poly-brominated diphenyl ether PBDE 73 (right).<smiles>NC(Cc1cc(I)c(Oc2cc(I)c(O)c(I)c2)c(I)c1)C(=O)O</smiles>

T4<smiles>NC(Cc1cc(I)c(Oc2ccc(O)c(I)c2)c(I)c1)C(=O)O</smiles>

T3<smiles>Oc1c(Br)cc(Oc2c(Br)cccc2Br)cc1Br</smiles>

PBDE 73

Biosensor formats for $\mathbf{T} 4$ generally make use of its natural receptors [12], but invariably need some form of signal amplification or competitive assay format, thus not complying always with the biosensor requirements [14]. With Surface Plasmon Resonance (SPR), the binding of T4 and its tri-iodinated analogue $\mathbf{T 3}$ can be studied in real time without the need for labeling [15].

Molecular imprinting is presently a broad field of research holding very high prospects for the detection of molecular targets, particularly drug substances [16,17]. The method is based on the templating effect of target molecules in a solidifying matrix (e.g., by polymerizing, freezing, or adsorbing/packing), as conducted in a suitable medium. The template is then removed from the matrix, expectedly leaving binding pockets selective for the target molecule. Imprinting is mostly achieved with cross-linked polymers as a matrix, but a viable alternative is represented by using the imprinted self-assembled monolayers ( $i$-SAMs), i.e., employing non-covalent interactions to assemble a surface layer in the presence of the templating molecule. Although the detection of small molecules with SPR may be cumbersome, a successful imprinting study was recently published on morphine [18].

The present work constitutes a preliminary study on the SPR detection of T4 by depositing self-assembled, T4-imprinted thin layers on gold surfaces. In order to achieve this goal, we designed a series of pyridine-modified derivatives of lipoic acid (see Figure 2). All the ligands are expected to establish hydrogen and halogen bonds with T4 in different measures. Their amide (1-5), pyridyl (2-4) and hydroxyl (5) groups are, in fact, capable of hydrogen bonding with the phenolic hydroxyl, amine, and carboxyl moieties of T4. The pyridyl derivatives $\mathbf{2 - 4}$, in particular, were designed to also enable short contacts with the iodine atoms of T4. Halogen bonding based on iodoarene-pyridyl couples has been reported to have a binding strength comparable to that of phenol-pyridine hydrogen bonds [19], and generally higher than that of iodine-oxygen interactions such as those inferred from TTR-thyroxine structures [8]. In principle, a weak halogen bond involving the amidic oxygen is also possible. The control compound $\mathbf{1}$ lacks the pyridyl moiety, but is more likely to engage in hydrophobic interactions, indicated by its higher $\log P$ value (see Experimental Section). Compound 5 is a ligand that was previously studied in $i$-SAM formation for morphine [18], and was employed here for comparison purposes. 
Figure 2. Lipoic acid derivatives used as ligands in the present work. Proton positions are labeled for NMR assignments.

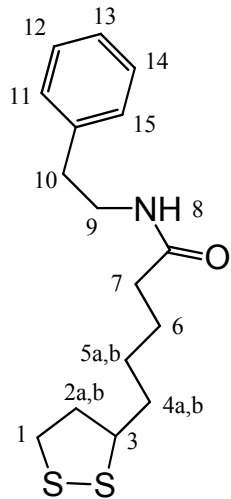

1

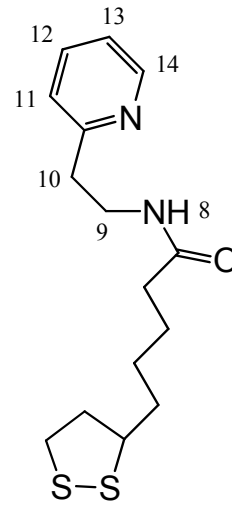

2

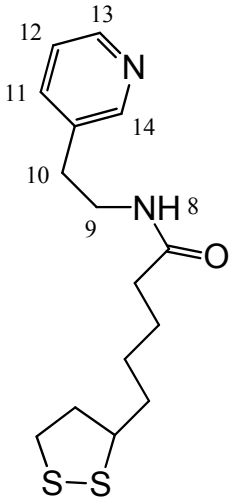

3

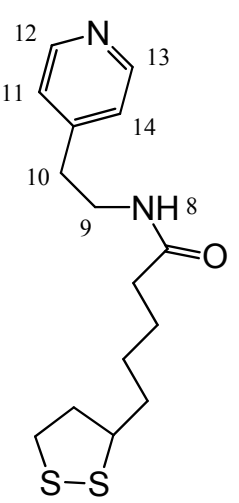

4

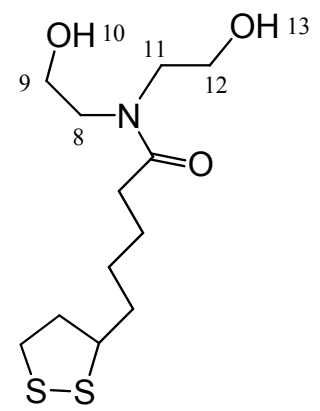

5

\section{Results and Discussion}

\subsection{Self-Assembly of the Lipoates and Thin Layer Formation}

A large set of SPR data was acquired to obtain information about the thickness of the layers assembled from compounds 1-4. The layers were prepared from 2 to $3 \mathrm{mM}$ solutions of the ligands in $70 \%$ ethanol, according to the earlier work [18]. In particular, the two-medium method appeared to give useful estimates of the thickness and refractive index of the thin layers [20]. This method comprises the measurement of SPR shifts in two media (in this case air and 70\% ethanol) and fitting of the SPR curves to theory in order to give an estimate for the thickness $(d)$ and refractive index $(n)$ of the layer. As an example, fitted SPR curves are given in Figure 3 for one of the measurements on ligand 4 as obtained with the SPR-Navi 200 spectrometer (Bionavis Ltd, Tampere, Finland). The angular shifts in air and 70\% ethanol for all the compounds are given in Figure S1 and an example of the fitting contour plots for $n$ and $d$ are given in Figure S2 of the supporting information.

Figure 3. SPR curves of incident light angle $(\theta)$ versus reflection coefficient $(R)$, measured in (A) air and (B) 70\% ethanol. Red curves were obtained on blank gold, while blue curves were acquired after layer formation of lipoate 4 . Fitted data are represented by the dotted curves.
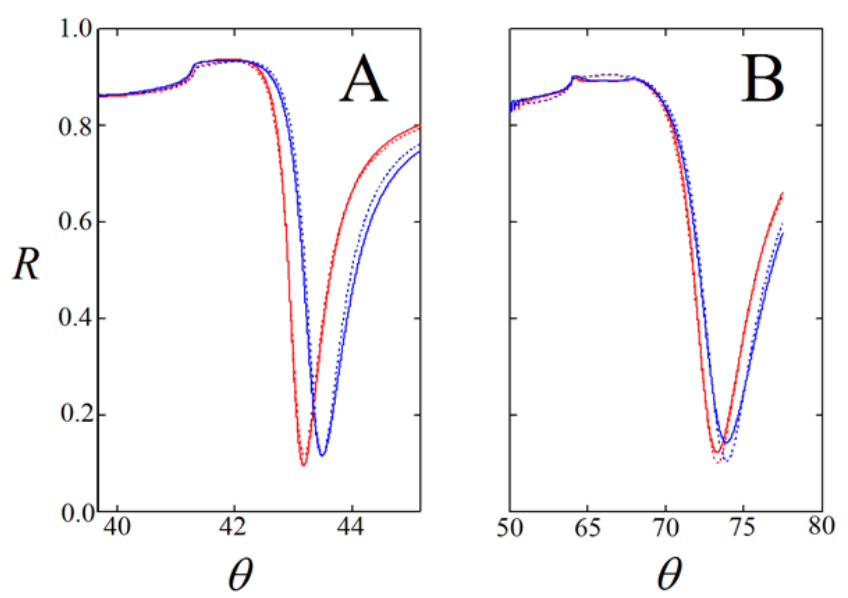
Table 1 summarizes the results for the thickness and refractive index (with standard error limits) of the deposited layers. Additionally, the density was calculated from the obtained refractive index using an estimated value of the molecular refractivity, while the surface coverage was subsequently calculated from the thickness and density values. These results indicated that the thickness of the layers was much more than a monolayer when the assembly was performed at 2-3 $\mathrm{mM}$ ligand concentration, particularly for compounds 2 and $\mathbf{3}$. The density and the refractive index of the layers were slightly lower than the calculated values (see Experimental Section), thus indicating not very tightly packed layers, which is credible considering that the dithiolane ring needs more space on the surface than linear alkylmercaptans. The density of the lipoate layers appeared to be largely the same $\left(1.0 \mathrm{~g} / \mathrm{cm}^{3}\right)$.

Table 1. Physicochemical constants of the ligands 1-5 and T4 assembled on clean gold in $70 \%$ EtOH from a concentration of $2 \mathrm{mM}(\mathbf{T} 4,5)$ or $3 \mathrm{mM}(1-4)$ : Molecular weight $\left(M_{r}\right)$, molar refractivity $(A)$, refractive index $(n)$, layer thickness $(d)$, density $(\rho)$, surface coverage $(\Gamma)$, and fractional surface coverage $(\Phi)$.

\begin{tabular}{cccccccccccc}
\hline Compound & $\boldsymbol{M}_{\boldsymbol{r}}$ & $\boldsymbol{A}^{\mathbf{a}}$ & \multicolumn{3}{c}{$\boldsymbol{n}^{\mathbf{b}}$} & \multicolumn{2}{c}{$\boldsymbol{d}(\mathbf{n m})$} & \multicolumn{2}{c}{$\boldsymbol{\rho}\left(\mathbf{g} / \mathbf{c m}^{\mathbf{3}}\right)^{\mathbf{c}}$} & $\boldsymbol{\Gamma}\left(\mathbf{n g} / \mathbf{c m}^{\mathbf{2}}\right)$ & $\boldsymbol{\Phi}^{\mathbf{d}}$ \\
\hline 1 & 309.49 & 90.8 & 1.48 & \pm 0.04 & 2.2 & \pm 0.4 & 0.96 & \pm 0.06 & 221 & \pm 33 & 1.6 \\
2 & 310.48 & 88.9 & 1.51 & \pm 0.02 & 4.2 & \pm 0.3 & 1.04 & \pm 0.04 & 451 & \pm 16 & 3.2 \\
3 & 310.48 & 88.9 & 1.47 & \pm 0.01 & 5.7 & \pm 0.13 & 0.99 & \pm 0.01 & 560 & \pm 11 & 4.0 \\
4 & 310.48 & 88.9 & 1.51 & \pm 0.02 & 3.1 & \pm 0.23 & 1.05 & \pm 0.03 & 322 & \pm 14 & 2.3 \\
5 & 293.45 & 78.7 & 1.47 & \pm 0.02 & 1.8 & \pm 0.1 & 1.03 & \pm 0.04 & 180 & \pm 4 & 1.8 \\
$\mathrm{~T} 4$ & 776.87 & 123.0 & 1.47 & \pm 0.02 & 1.9 & \pm 0.4 & 1.75 & \pm 0.11 & 320 & \pm 95 & - \\
\hline
\end{tabular}

${ }^{\mathrm{a}}$ The molar refractivity $A$ and $\log \mathrm{P}$ values were estimated using software from ADC Labs [21]; ${ }^{\mathrm{b}}$ Standard error $=\sigma / \sqrt{ } \mathrm{n} .{ }^{\mathrm{c}}$ Density of the layer was calculated from the values of $M_{r}, R_{m}$, and $n$ according to the relation: $\rho=\left(n^{2}-1\right) /\left(n^{2}+2\right) \cdot M_{r} / A .{ }^{\mathrm{d}}$ Fractional surface coverage is the amount of monolayers, based on the estimated surface coverage of a monolayer.

T4 itself showed substantial layer formation onto the gold surface, and the observed refractive index was significantly smaller than the theoretical value (1.80). This might indicate that the layer includes solvent molecules. As can be reasonably expected, the density of the $\mathbf{T 4}$ layer was higher $\left(1.75 \mathrm{~g} / \mathrm{cm}^{3}\right)$ than that of the lipoates, but it was much lower than the theoretical value (2.64). Based on these results it can be postulated that $\mathbf{2}$ and $\mathbf{3}$ may have some tendency to dimerize by interaction between the pyridine and the amide hydrogen, and thus form thicker layers compared to 4 . The overall surface area per molecule of monolayers of the ligands was estimated by molecular calculations [22-24], and were approximately $37 \AA^{2}$ per molecule for the pyridyl and phenyl ligands (1-4) and $48 \AA^{2}$ per molecule for ligand 5, yielding a surface coverage of $140 \mathrm{ng} / \mathrm{cm}^{2}$ and $100 \mathrm{ng} / \mathrm{cm}^{2}$, respectively. Thus, multilayers were formed at the conditions used, as indicated by the fractional surface coverage in Table 1.

\subsection{Binding of $\boldsymbol{T} 4$ to Self-Assembled Lipoate Layers}

Initially, the binding of T4 to the layers of ligands 1-4 on gold was studied with SPR using the Biacore 3000 instrument in phosphate buffered saline solution with $0.1 \% \mathrm{DMSO}$ and $\mathrm{pH}=9.0$. High $\mathrm{pH}$ and DMSO were initially used to increase the solubility of T4. The self-assembly of ligands 1-4 
was performed here ex situ before each binding experiment in $70 \%$ ethanol, which was a suitable solvent for all compounds used. Results were fitted to the Langmuir-Freundlich isotherm (see Experimental Section) to estimate the affinity constant $\left(K_{\mathrm{A}}\right)$, the binding capacity $(Q)$, and the binding exponent $(v)$.

The observed binding curves of T4 were quite different for the various surfaces tested (Figure 4), and showed a marked step-wise response (with $v>1$ ) at a particular concentration for blank gold $\left(K_{\mathrm{A}} \approx 7 \times 10^{7}\right)$ and compounds $1\left(K_{\mathrm{A}} \approx 4 \times 10^{6}\right)$ and $\mathbf{3}\left(K_{\mathrm{A}} \approx 2 \times 10^{7}\right)$, while rather heterogeneous binding $(v<1)$ was evidenced for the compounds $2\left(K_{\mathrm{A}} \approx 3 \times 10^{7}\right)$ and $4\left(K_{\mathrm{A}} \approx 7 \times 10^{5}\right)$. Although compound $\mathbf{4}$ had the lowest affinity, it showed the largest binding capacity for T4 ( $Q=1360 \mathrm{RU})$. Thus, rather characteristic differences were observed for the pyridyl-lipoate conjugates without imprinting. The T4 adsorption level on the self-assembled layer of compound $\mathbf{1}$ (the control) was lowest: this appears to indicate that the expected higher tendency of ligand $\mathbf{1}$ to form hydrophobic interactions with $\mathbf{T} 4$ cannot make up for the affinity loss caused by the lack of a pyridyl group capable of establishing hydrogen- and halogen bonds with T4. As compound 4 displayed the largest binding capacity, and has the pyridine nitrogen on a site that is more amenable to hydrogen and halogen bonding, it emerged as the most promising candidate for further study with imprinted layers. The strong affinity binding of $\mathbf{T} 4$ for blank gold, however, should be taken in consideration as a possible obstacle to imprinting by self-assembly, because it may be difficult to elute the template from the layer.

Figure 4. Adsorption isotherms of T4 on clean gold (- - ) and onto self-assembled layers of the pyridyl-lipoate conjugates $1(--), 2(--), 3(--)$, and $4(--)$. Measurements were performed in PBS $(150 \mathrm{mM} \mathrm{NaCl}, 50 \mathrm{mM}$ phosphate buffer, $\mathrm{pH}=9.0)$ supplemented with $0.1 \%$ DMSO.

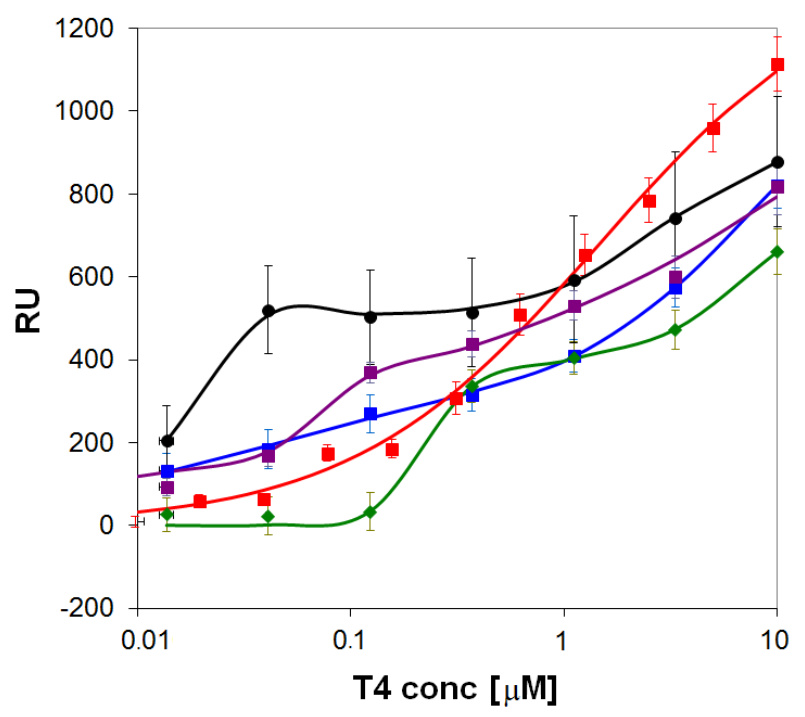

\subsection{Imprinted Self-Assembled Layers with $\mathbf{T 4}$}

In order to achieve suitable conditions for layers formation that enable the observation of imprinting effects, preliminary experiments were performed with mixtures of $\mathbf{T 4}$ as template molecule and compound 4 as ligand. The mixtures were also made in 70\% ethanol and manually spotted (ex situ) on a peroxide-cleaned gold chip. The coated chip was mounted into the SPR instrument (Biacore 3000) 
and was subjected to an elution step, to remove $\mathbf{T} 4$ from the layer (either with high or low $\mathrm{pH}$ buffer solutions). Hereafter, rebinding of $\mathbf{T} 4$ was studied in aqueous buffer solutions (either PBS or HBS at $\mathrm{pH}=7.4$ ). The rebinding was performed at a single concentration of $\mathbf{T} \mathbf{4}$, or at a broad range of concentrations, constructing a titration curve.

The solvent for layer formation (70\% ethanol) was chosen for its ability to dissolve both T4 and 4 . However, such solvent was damaging the fluidics system of the Biacore 3000 instrument, and thus, in some cases, in situ layer formation in this solvent was measured separately with another SPR instrument in $70 \%$ EtOH (the SPR-Navi 200) (a screen shot of an in situ measurement is given in the supporting information, Figure S3). It was observed that mixtures of T4 and $\mathbf{4}$ gave a somewhat lower SPR response compared to that of the pure compounds, indicating formation of slightly thinner (or less dense) layers for the mixtures than for the pure compounds (Figure 5).

Figure 5. The SPR angle shift as a function of molar fraction of T4 in a mixture with ligand 4. The data points are given in blue, and the averages (with error bars) plotted in black. At molar fraction 0.0 the SPR shift is due to pure ligand 4, while at fraction 1.0 the SPR shift is due to pure $\mathbf{T} 4$.

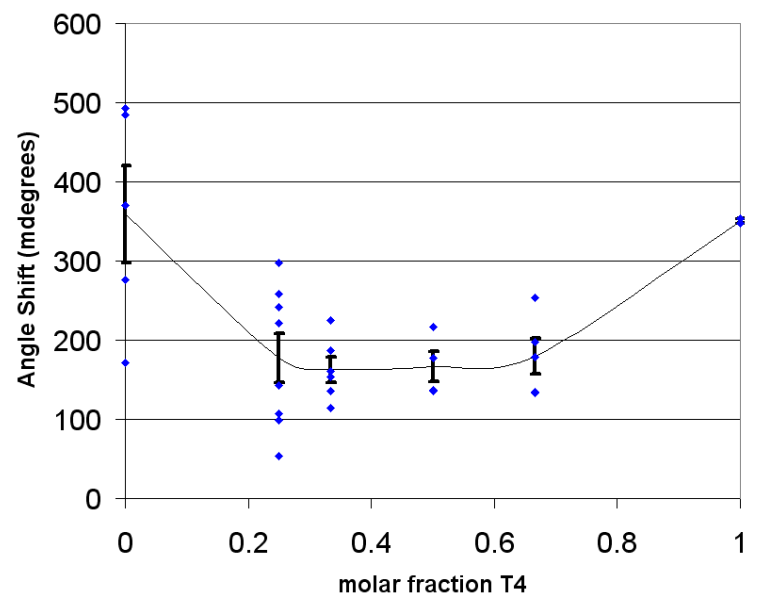

Since mixing of T4 with $\mathbf{4}$ had a significant effect on the layer formation, the first tests with ex situ imprinted layers were made at various molar ratios of $\mathbf{4}$ with $\mathbf{T 4}$, also with reference to ligand $\mathbf{5}$ as studied in the earlier work [18]. In the various trials, the binding of T4 to binary layers of $\mathbf{T 4}$ and ligand 4 was studied initially in PBS buffer, using various regeneration reagents (see Figure S4). It was noticed that denser (or thicker) layers were produced with ligand $\mathbf{4}$ in comparison with ligand $\mathbf{5}$, and that there was a maximum in layer thickness for the molar ratio (T4/4) of 1:2. However, PBS buffer gave rise to a decreasing trend in repeated elution/rebinding tests, while stable regeneration cycles could be attained with HBS buffer $(\mathrm{pH}=6.8)$. This was evidenced on layers formed ex situ from $1.0 \mathrm{mM} \mathrm{T4}$ and $2.0 \mathrm{mM} 4$ in $70 \%$ ethanol, using $0.1 \mathrm{M} \mathrm{NaOH}$ as the regeneration reagent (Figure 6) in HBS buffer. Thus, elution and rebinding of $\mathbf{T} 4$ on imprinted layers of $\mathbf{4}$ and $\mathbf{5}$ were studied further using similar conditions as in Figure 6, employing titrations of T4 after each elution step. As far as regeneration conditions are concerned, treatment with strong alkaline solution appeared to be more effective than treatment with $1 \mathrm{M} \mathrm{HCl}$. This can be easily explained by the enhanced solubility of T4 at higher $\mathrm{pH}$, when de-protonation of the phenolic hydroxyl occurs. 
Figure 6. SPR response to five cycles of elution/rebinding of a binary layer of T4 $(1 \mathrm{mM})$ and $4(2 \mathrm{mM})$, using HBS running buffer. The regeneration agent was $0.1 \mathrm{M} \mathrm{NaOH}$ and the T4 standard was set at $3.3 \mu \mathrm{M}$ in HBS buffer. The negative SPR shift of the regeneration step was plotted in positive direction to allow comparison with the rebinding of $\mathbf{T 4}$.

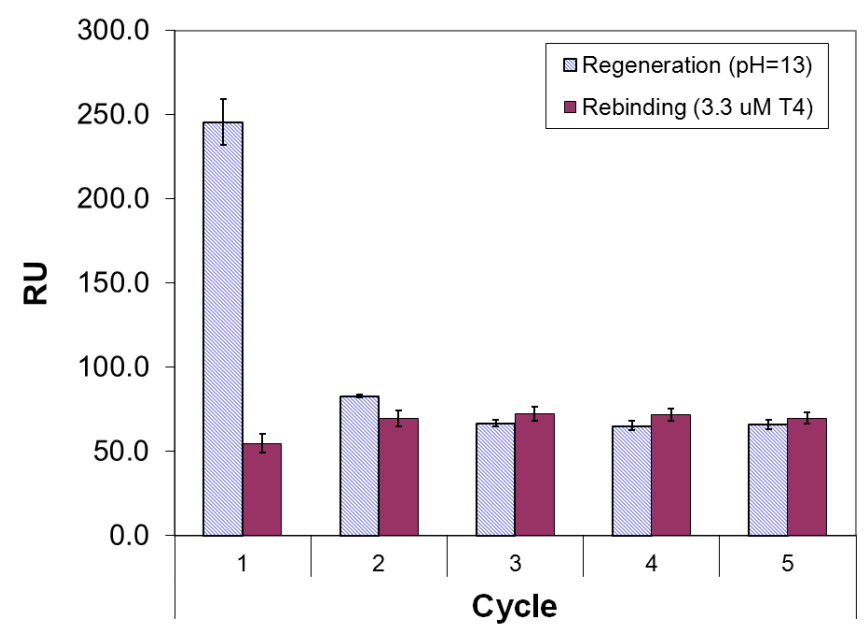

When 2 mM 4 was mixed with $0.5 \mathrm{mM} \mathrm{T4}$ and spotted ex situ onto clean gold, a total film thickness of $11,700( \pm 170) \mathrm{RU}$ was attained (in 70\% EtOH), which represents a much thicker multilayer of T4 and 4. The first elution with $0.1 \mathrm{M} \mathrm{NaOH}$ caused a dissociation of $1790( \pm 140) \mathrm{RU}$, and subsequent elution steps after the titrations also resulted in rather substantial dissociation (Figure 7A). Titration with T4 in the concentration range $0.014-10 \mathrm{M}$ between these elution steps produced only minor binding initially, but upon repeated elution significant rebinding of $\mathbf{T 4}$ started to occur (Figure 7B). The binding constants of the fifth cycle were: $K_{\mathrm{A}}=1.4 \times 10^{6} \mathrm{M}^{-1}, Q=475 \mathrm{RU}$ and $v=0.29$, indicating a low affinity heterogeneous rebinding process. The binding observed here was lower than the one found for $\mathbf{T 4}$ on non-imprinted ligand $\mathbf{4}$ layers. Although these two binding experiments were performed in somewhat different conditions and are therefore not directly comparable, this may be an indication of a non-optimal efficiency of the regeneration of the imprinted layers, in which a significant amount of the binding sites would remain occupied by the $\mathbf{T} 4$ molecules.

Similar experiments conducted with compound $\mathbf{5}$ and with a mixture of $\mathbf{4}$ and $\mathbf{5}$ yielded similar characteristics: an increasing response of the layer upon elution (see Figure S5). These results indicated that by using ligand $\mathbf{4}$ it was indeed possible to obtain rebinding, albeit at a relatively low affinity. For ligand 5 the binding constants obtained were $K_{\mathrm{A}}=5.6 \times 10^{4} \mathrm{M}^{-1}, Q=678 \mathrm{RU}$ and $v=0.25$, which signifies a lower affinity and higher degree of heterogeneity compared to ligand 4 . The lower affinity value may also be due to ligand $\mathbf{5}$ being more hydrophilic than $\mathbf{4}$ and therefore less prone towards hydrophobic interactions, although these already appeared to play a secondary role in the binding of $\mathbf{T} 4$ to non-imprinted layers. Use of both ligands $\mathbf{4}$ and $\mathbf{5}$ in molar ratio 1:1 for the formation of an imprinted layer yielded the following binding constant: $K_{\mathrm{A}}=2.7 \times 10^{6} \mathrm{M}^{-1}, Q=220 \mathrm{RU}$ and $v=0.39$. Thus, a minor synergistic effect of the two ligands could be discerned, producing a higher affinity and slightly lower heterogeneity compared to the use of ligand 4 alone. 
Figure 7. (A) Amount of complex desorbed upon successive elution steps with $0.1 \mathrm{M}$ $\mathrm{NaOH}$; (B) Rebinding of T4 after elution. The running buffer was HBS.
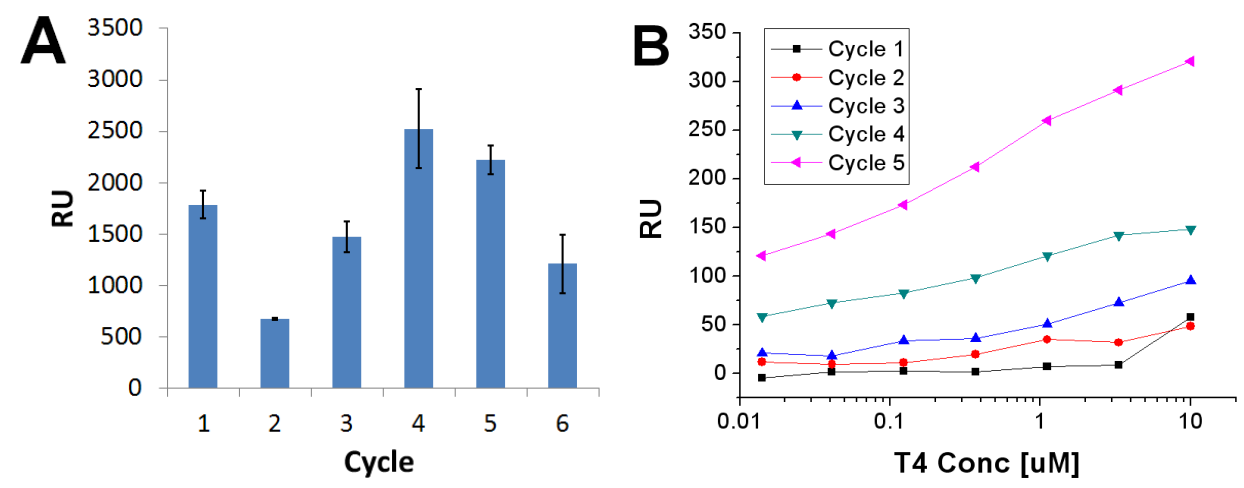

\section{Experimental Section}

\subsection{Materials and Methods}

For preparation of the ligands, lipoic acid, phenethylamine, 2-(2-aminoethyl)-pyridine, 4-(2-aminoethyl)-pyridine or 3-(2-aminoethyl)pyridine hydrobromide, and hydroxysuccinimide were obtained from Fluka and Aldrich. The ethanol used in this study was AA grade from Altia (Rajamäki, Finland). Other solvents used were DMSO (Baker). Buffer solutions were PBS (150 $\mathrm{mM} \mathrm{NaCl}$, $10 \mathrm{mM}$ phosphate $\mathrm{pH}=7.45)$ or HBS (150 $\mathrm{mM} \mathrm{NaCl}, 10 \mathrm{mM}$ HEPES $\mathrm{pH}=6.8)$.

${ }^{1} \mathrm{HNMR}$ spectra were recorded in $\mathrm{CDCl}_{3}$ on a Varian Oxford 300 instrument $(300 \mathrm{MHz})$. Electro-spray mass spectrometry (ESI-MS) was performed on a LTQ Orbitrap XL spectrometer (Thermo Scientific, Waltham, MA, USA) in positive mode. For the characterization of compound 5 a different MS instrument was used: a Bruker MicrOTOF-Q in positive ion electrospray mode.

Binding studies in 70\% ethanol were performed on a SPR-Navi 200 instrument (BioNavis Ltd., Ylöjärvi, Finland), which records whole SPR spectra (reflectivity vs. angle), while in aqueous environment the binding was predominantly studied with the Biacore 3000 instrument (Biacore Life Sciences, Uppsala, Sweden). These instruments have different measurement wavelengths (SPR-Navi at $670 \mathrm{~nm}$, Biacore 3000 at $760 \mathrm{~nm}$ ), and thus the results are not directly comparable. For the SPR-Navi the measured unit was in degrees or millidegrees of angular shift of the SPR minimum. The measured parameter for the Biacore 3000 was the "resonance unit" (RU). 1 RU represents a $10^{-4}$ degree angular shift of the SPR minimum, and for common biomolecules corresponds to a surface coverage of $1 \mathrm{pg} / \mathrm{mm}^{2}$ [25]. This estimate may be applied to the lipoate layers, which have similar refractive index as biomolecules, but not to $\mathbf{T} 4$ due to its markedly higher molar refractivity.

\subsection{Preparation of Lipoamide Derivatives}

In a typical synthesis, lipoic acid $(1.47 \mathrm{mmol})$ and $N$-hydroxy-succinimide $(1.61 \mathrm{mmol})$ were dissolved in $10 \mathrm{~mL}$ of THF. Dicyclohexylcarbodiimide $(1.75 \mathrm{mmol})$ was added, and precipitation occurred within few minutes. The mixture was stirred overnight at room temperature, and the solid precipitate was filtered off. 
According to cases, phenethylamine, 2-(2-aminoethyl)pyridine, 4-(2-aminoethyl)-pyridine or 3-(2-aminoethyl)pyridine dihydrobromide $(1.59 \mathrm{mmol})$ was added (note: for the hydrobromide salt, also $11.3 \mathrm{mmol}$ sodium bicarbonate and $5 \mathrm{ml}$ distilled water were added), and the mixture was stirred overnight at room temperature. The solvent was evaporated on a rotation evaporator, and addition of $15 \mathrm{~mL}$ dichloromethane allowed partial re-dissolution of the solid. The precipitate was filtered off. The solution was treated three times with $10 \mathrm{~mL}$ of saturated $\mathrm{KCl}$ aqueous solution, and the organic phase was dried on anhydrous sodium sulphate and filtered on paper.

Purification was performed by column chromatography using $5 / 1$ or $10 / 1 \quad(v / v)$ dichloromethane/ethanol mixtures as an eluent, according to cases. Pure products were recovered in about 55\% yield for pyridyl derivatives, and in 70\% yield for the phenyl derivative. Solubility of the ligands and thyroxine was generally low in aqueous solutions, but fairly good in $70 \%$ ethanol at the used concentrations for imprinting $(10 \mathrm{mg} / \mathrm{mL}$ stock).

Characterization of the compounds was as follows. Theoretical values of the $\log P$ (the octanol/water distribution coefficient, a measure of the hydrophobicity of the compound), molecular refractivity $(A)$, refractive index $(n)$ and density $(\rho)$ were calculated with the ACD/Labs software [22-24].

5-(1,2-Dithiolan-3-yl)-N-[(2-phenyl-ethyl)]pentanamide (1, phenylethyl lipoamide, $\left.M_{\mathrm{r}}=309.49\right)$. ${ }^{1} \mathrm{HNMR}\left(\mathrm{CDCl}_{3}\right)$ : $\delta$ 7.34-7.18 (m, 5H; $\mathrm{H}_{11}$ to $\left.\mathrm{H}_{15}\right) ; 5.40\left(\right.$ br s, $\left.1 \mathrm{H} ; \mathrm{H}_{8}\right) ; 3.57-3.49\left(\mathrm{~m}, 3 \mathrm{H} ; \mathrm{H}_{3}, \mathrm{H}_{9}\right)$; $3.18-3.09\left(\mathrm{~m}, 2 \mathrm{H} ; \mathrm{H}_{1}\right) ; 2.82\left(\mathrm{t}, 2 \mathrm{H},{ }^{3} J_{H, H}=7.2 \mathrm{~Hz} ; \mathrm{H}_{10}\right) ; 2.45\left(\mathrm{~m}, 1 \mathrm{H} ; \mathrm{H}_{2 \mathrm{a}}\right) ; 2.12\left(\mathrm{t}, 2 \mathrm{H},{ }^{3} J_{H, H}=6.9 \mathrm{~Hz}\right.$; $\left.\mathrm{H}_{7}\right) ; 1.90\left(\mathrm{~m}, 1 \mathrm{H} ; \mathrm{H}_{2 \mathrm{~b}}\right), 1.64\left(\mathrm{~m}, 4 \mathrm{H} ; \mathrm{H}_{4 \mathrm{a}}, \mathrm{H}_{5 \mathrm{a}}, \mathrm{H}_{6}\right), 1.44\left(\mathrm{~m}, 2 \mathrm{H} ; \mathrm{H}_{4 \mathrm{~b}}, \mathrm{H}_{5 \mathrm{~b}}\right) . \mathrm{MS}(\mathrm{ESI})(\mathrm{M}+\mathrm{H})^{+}$: found 310.12834. Physical properties: $\log \mathrm{P}=3.57 \pm 0.42 ; R_{m}=90.8 \pm 0.3 \mathrm{~cm}^{3} ; n=1.58 \pm 0.02$; $\rho=1.13 \pm 0.06 \mathrm{~g} / \mathrm{cm}^{3}$.

5-(1,2-Dithiolan-3-yl)-N-[2-(pyridin-2-yl)ethyl]pentanamide (2, 2-pyridylethyl lipoamide, $\left.M_{\mathrm{r}}=310.48\right)$. ${ }^{1} \mathrm{HNMR}\left(\mathrm{CDCl}_{3}\right): \delta 8.53\left(\mathrm{~d}, 1 \mathrm{H},{ }^{3} J_{H, H}=4.8 \mathrm{~Hz} ; \mathrm{H}_{14}\right) ; 7.61\left(\mathrm{t}, 1 \mathrm{H},{ }^{3} J_{H, H}=8.1 \mathrm{~Hz} ; \mathrm{H}_{12}\right) ; 7.16(\mathrm{~d}, 1 \mathrm{H}$, $\left.{ }^{3} J_{H, H}=6.3 \mathrm{~Hz} ; \mathrm{H}_{11}\right) ; 7.15\left(\mathrm{t}, 1 \mathrm{H},{ }^{3} J_{H, H}=6.0 \mathrm{~Hz} ; \mathrm{H}_{13}\right) ; 6.41\left(\mathrm{br} \mathrm{s}, 1 \mathrm{H} ; \mathrm{H}_{8}\right) ; 3.67\left(\mathrm{dt}, 2 \mathrm{H},{ }^{3} J_{H, H}=6.0 \mathrm{~Hz}\right.$; $\left.\mathrm{H}_{9}\right) ; 3.54\left(\mathrm{~m}, 1 \mathrm{H} ; \mathrm{H}_{3}\right) ; 3.20-3.05\left(\mathrm{~m}, 2 \mathrm{H} ; \mathrm{H}_{1}\right) ; 2.98\left(\mathrm{t}, 2 \mathrm{H},{ }^{3} J_{H, H}=6.6 \mathrm{~Hz} ; \mathrm{H}_{10}\right) ; 2.43\left(\mathrm{~m}, 1 \mathrm{H} ; \mathrm{H}_{2 \mathrm{a}}\right) ; 2.15$ $\left(\mathrm{t}, 2 \mathrm{H},{ }^{3} J_{H, H}=7.5 \mathrm{~Hz} ; \mathrm{H}_{7}\right) ; 1.89\left(\mathrm{~m}, 1 \mathrm{H} ; \mathrm{H}_{2 \mathrm{~b}}\right) ; 1.66\left(\mathrm{~m}, 4 \mathrm{H} ; \mathrm{H}_{4 \mathrm{a}}, \mathrm{H}_{5 \mathrm{a}}, \mathrm{H}_{6}\right) ; 1.45\left(\mathrm{~m}, 2 \mathrm{H} ; \mathrm{H}_{4 \mathrm{~b}}, \mathrm{H}_{5 \mathrm{~b}}\right) . \mathrm{MS}$ (ESI) $(\mathrm{M}+\mathrm{H})^{+}$: found 311.12325. Physical properties: $\operatorname{LogP}=2.07 \pm 0.43 ; R_{m}=88.9 \pm 0.3 \mathrm{~cm}^{3}$; $n=1.58 \pm 0.02 ; \rho=1.16 \pm 0.06 \mathrm{~g} / \mathrm{cm}^{3}$.

5-(1,2-Dithiolan-3-yl)-N-[2-(pyridin-3-yl)ethyl]pentanamide (3, 3-pyridylethyl lipoamide, $\left.M_{\mathrm{r}}=310.48\right)$. ${ }^{1} \mathrm{HNMR}\left(\mathrm{CDCl}_{3}\right):{ }^{1} \mathrm{HNMR}\left(\mathrm{CDCl}_{3}\right): \delta 8.47\left(\mathrm{~d}, 1 \mathrm{H},{ }^{3} J_{H, H}=4.5 \mathrm{~Hz} ; \mathrm{H}_{13}\right) ; 8.44\left(\mathrm{~s}, 1 \mathrm{H} ; \mathrm{H}_{14}\right) ; 7.53(\mathrm{~d}, 1 \mathrm{H}$, $\left.{ }^{3} J_{H, H}=7.8 \mathrm{~Hz} ; \mathrm{H}_{11}\right) ; 7.24\left(\mathrm{dd}, 1 \mathrm{H},{ }^{3} J_{H, H}=4.8\right.$ and $\left.7.5 \mathrm{~Hz} ; \mathrm{H}_{12}\right) ; 5.57\left(\right.$ br s, $\left.1 \mathrm{H} ; \mathrm{H}_{8}\right) ; 3.60-3.49(\mathrm{~m}, 3 \mathrm{H}$; $\left.\mathrm{H}_{3}, \mathrm{H}_{9}\right) ; 3.21-3.06\left(\mathrm{~m}, 2 \mathrm{H} ; \mathrm{H}_{1}\right) ; 2.83\left(\mathrm{t}, 2 \mathrm{H},{ }^{3} J_{H, H}=6.9 \mathrm{~Hz} ; \mathrm{H}_{10}\right) ; 2.47\left(\mathrm{~m}, 1 \mathrm{H} ; \mathrm{H}_{2 \mathrm{a}}\right) ; 2.14(\mathrm{t}, 2 \mathrm{H}$, $\left.{ }^{3} J_{H, H}=6.9 \mathrm{~Hz} ; \mathrm{H}_{7}\right) ; 1.91\left(\mathrm{~m}, 1 \mathrm{H} ; \mathrm{H}_{2 \mathrm{~b}}\right) ; 1.66\left(\mathrm{~m}, 4 \mathrm{H} ; \mathrm{H}_{4 \mathrm{a}}, \mathrm{H}_{5 \mathrm{a}}, \mathrm{H}_{6}\right) ; 1.44\left(\mathrm{~m}, 2 \mathrm{H} ; \mathrm{H}_{4 \mathrm{~b}}, \mathrm{H}_{5 \mathrm{~b}}\right) . \mathrm{MS}(\mathrm{ESI})$ $(\mathrm{M}+\mathrm{H})^{+}$: found 311.12360. Physical properties: $\log \mathrm{P}=2.07 \pm 0.43 ; R_{m}=88.9 \pm 0.3 \mathrm{~cm}^{3}$; $n=1.58 \pm 0.02 ; \rho=1.16 \pm 0.06 \mathrm{~g} / \mathrm{cm}^{3}$.

5-(1,2-Dithiolan-3-yl)-N-[2-(pyridin-4-yl)ethyl]pentanamide (4, 4-pyridylethyl lipoamide, $\left.M_{\mathrm{r}}=310.48\right)$. ${ }^{1} \mathrm{HNMR}\left(\mathrm{CDCl}_{3}\right): \delta 8.53\left(\mathrm{~d}, 2 \mathrm{H} ;{ }^{3} J_{H, H}=4.5 \mathrm{~Hz} ; \mathrm{H}_{12}, \mathrm{H}_{13}\right) ; 7.13\left(\mathrm{~d}, 2 \mathrm{H},{ }^{3} J_{H, H}=4.2 \mathrm{~Hz} ; \mathrm{H}_{11}, \mathrm{H}_{14}\right) ; 5.42$ (br s, $\left.1 \mathrm{H} ; \mathrm{H}_{8}\right) ; 3.60-3.49\left(\mathrm{~m}, 3 \mathrm{H} ; \mathrm{H}_{3}, \mathrm{H}_{9}\right) ; 3.22-3.07\left(\mathrm{~m}, 2 \mathrm{H} ; \mathrm{H}_{1}\right) ; 2.84\left(\mathrm{t}, 2 \mathrm{H},{ }^{3} J_{H, H}=6.6 \mathrm{~Hz} ; \mathrm{H}_{10}\right)$; $2.45\left(\mathrm{~m}, 1 \mathrm{H} ; \mathrm{H}_{2 \mathrm{a}}\right) ; 2.14\left(\mathrm{t}, 2 \mathrm{H},{ }^{3} J_{H, H}=6.6 \mathrm{~Hz} ; \mathrm{H}_{7}\right) ; 1.90\left(\mathrm{~m}, 1 \mathrm{H} ; \mathrm{H}_{2 \mathrm{~b}}\right) ; 1.73-1.41\left(\mathrm{~m}, 6 \mathrm{H} ; \mathrm{H}_{4 \mathrm{a}, \mathrm{b}}\right.$, 
$\left.\mathrm{H}_{5 \mathrm{a}, \mathrm{b}}, \mathrm{H}_{6}\right)$. MS (ESI) $(\mathrm{M}+\mathrm{H})^{+}$: found 311.12329. Physical properties: $\log \mathrm{P}=2.07 \pm 0.43$; $R_{m}=88.9 \pm 0.3 \mathrm{~cm}^{3} ; n=1.58 \pm 0.02 ; \rho=1.13 \pm 0.06 \mathrm{~g} / \mathrm{cm}^{3}$.

5-(1,2-Dithiolan-3-yl)-N,N-bis(2-hydroxy-ethyl)-pentanamide (5, di-(2-hydroxyethylamino)-lipo-amide, $\left.M_{\mathrm{r}}=293.45\right) .{ }^{1} \mathrm{HNMR}\left(\mathrm{CDCl}_{3}\right): \delta 3.93\left(\right.$ br s, $\left.2 \mathrm{H}, \mathrm{H}_{10}, \mathrm{H}_{13}\right) ; 3.81\left(\mathrm{t}, 2 \mathrm{H},{ }^{3} J_{H, H}=4.8 \mathrm{~Hz} ; \mathrm{H}_{9}\right.$ or $\left.\mathrm{H}_{12}\right)$; $3.76\left(\mathrm{t}, 2 \mathrm{H},{ }^{3} J_{H, H}=4.7 \mathrm{~Hz}, \mathrm{H}_{9}\right.$ or $\left.\mathrm{H}_{12}\right) ; 3.57\left(\mathrm{~m}, 1 \mathrm{H}, \mathrm{H}_{3}\right) ; 3.52\left(\mathrm{t}, 2 \mathrm{H},{ }^{3} J_{H, H}=5.1 \mathrm{~Hz} ; \mathrm{H}_{8}\right.$ or $\left.\mathrm{H}_{11}\right) ; 3.48(\mathrm{t}$, $2 \mathrm{H},{ }^{3} J_{H, H}=5.2 \mathrm{~Hz} ; \mathrm{H}_{8}$ or $\left.\mathrm{H}_{11}\right) ; 3.13\left(\mathrm{~m}, 2 \mathrm{H} ; \mathrm{H}_{1}\right) ; 2.45\left(\mathrm{~m}, 1 \mathrm{H} ; \mathrm{H}_{2 \mathrm{a}}\right) ; 2.40\left(\mathrm{t}, 2 \mathrm{H},{ }^{3} J_{H, H}=7.5 \mathrm{~Hz} ; \mathrm{H}_{7}\right)$; $1.90\left(\mathrm{~m}, 1 \mathrm{H} ; \mathrm{H}_{2 \mathrm{~b}}\right) ; 1.66\left(\mathrm{~m}, 4 \mathrm{H} ; \mathrm{H}_{4 \mathrm{a}}, \mathrm{H}_{5 \mathrm{a}}, \mathrm{H}_{6}\right) ; 1.46\left(\mathrm{~m}, 2 \mathrm{H} ; \mathrm{H}_{4 \mathrm{~b}}, \mathrm{H}_{5 \mathrm{~b}}\right) . \mathrm{MS}(\mathrm{ESI})(\mathrm{M}+\mathrm{H})^{+}$: found 294.1267. Physical properties: $\operatorname{LogP}=1.07 \pm 0.38 ; R_{m}=78.7 \pm 0.3 \mathrm{~cm}^{3} ; n=1.57 \pm 0.02 ; \rho=1.220 \pm 0.06$.

\subsection{Preparation of the Imprinted Films and Titration Experiments}

The ex situ preparation of the imprinted films consisted of the following steps: (1) cleaning of a gold chip in boiling $\mathrm{NH}_{4} \mathrm{OH}(30 \%) / \mathrm{H}_{2} \mathrm{O}_{2}(30 \%) / \mathrm{H}_{2} \mathrm{O}(1: 1: 7 v / v)$; (2) rinse of the slide with fresh MilliQ-grade water and immersion in 70\% ethanol; (3) blowing dry the chip with a burst of inert gas and dispensing of $50-150 \mu \mathrm{L}$ of a mixture of template (T4) and ligand at specified molar ratio, allowing the self-assembly to take place in a small Petri dish for 1 hour in 70\% ethanol; (4) washing the slide with $70 \%$ ethanol and drying in a laminar flow cabinet in air, and (5) gluing the gold substrate in a Biacore cassette and inserting it in the Biacore 3000 instrument; (6) recording a baseline in buffer solution for at least $0.5 \mathrm{~h}$ until a stable baseline was obtained. Hereafter, elution solution $(0.1 \mathrm{M}$ $\mathrm{NaOH}$ ) was injected and the amount desorbed (in "resonance units", RU) recorded, and series of standards of T4 in buffer solution were injected to construct a titration curve. Various cycles were run to see if there was any change in the response as a result of regeneration. Titration curves were fitted to the Langmuir-Freundlich (or Sips) isotherm (Equation 1), in which $\mathrm{C}$ is the molar concentration of the adsorbing species, $R$ the SPR response and $Q$ is the binding capacity (maximum binding response, sometimes also denoted as $R_{\max }$ ), $K_{\mathrm{A}}$ the affinity constant, and $\mathbf{v}$ the binding exponent. $v=1$ indicates normal Langmuir binding, $v<1$ indicates heterogeneity of binding sites, while $v>1$ indicates cooperativity of binding [26].

$$
R=\frac{Q\left(K_{\mathrm{A}} C\right)^{v}}{1+\left(K_{\mathrm{A}} C\right)^{v}}
$$

The preparation of films onto gold was also studied in real time (in situ) with the SPR-Navi instrument by inserting a larger gold chip after peroxide cleaning into the instrument, and docking it to a 2-channel, temperature-controlled fluidic system as part of a typical FIA-configuration. A running solution of $70 \%$ ethanol was applied by use of a peristaltic pump at set at $20 \mu \mathrm{L} / \mathrm{min}$. The mixture of template and ligand was injected twice via an injection valve into the solvent stream. The angular shift can be followed in real time, but also the whole SPR curves can be recorded. The refractive index and thickness of the films were calculated with a recently developed method, which utilizes SPR spectra recorded in two media of known refractive index before and after film deposition [27]. The fitting algorithm utilized a theoretical model of the SPR phenomenon based on the Müller matrix representation of polarized light reflected from stratified thin optical layers [20]. 


\section{Conclusions}

The results indicated that the three pyridyl derivatives of lipoic acid showed multilayer formation at concentrations comparable to those of an earlier study (1-2 mM) [18]. The self-assembled layers of compounds 1-4 interacted with T4 layers in quite different fashion. The largest binding capacity was obtained for ligand 4 which showed an affinity constant of $K_{\mathrm{A}} \approx 7 \times 10^{5} \mathrm{M}^{-1}$. When depositing mixed layers of $\mathbf{T} 4$ and ligand $\mathbf{4}$ to assess the possibility of molecular imprinting, the best rebinding effects could be observed in HBS buffer ( $\mathrm{pH}=7.45$ ), attaining an affinity constant of $1.4 \times 10^{6} \mathrm{M}^{-1}$. A combination of ligand 4 with ligand 5 slightly increased the affinity $\left(K_{\mathrm{A}}=2.7 \times 10^{6} \mathrm{M}^{-1}\right)$. However, up to five regeneration cycles were required to obtain any response to $\mathbf{T 4}$, using a strong $0.1 \mathrm{M} \mathrm{NaOH}$ elution reagent. This is likely due to the removal of excess, weakly bound species, while stronger ligand-template interactions took place at sites in closer vicinity of the gold surface.

These results should however be considered preliminary, as a definitive confirmation of the imprinting effect would require the binding experiments to be performed in the same conditions as the imprinted layer formation. This could not be achieved in the present work, as the pyridyl ligands required deposition in ethanol, while binding/rebinding studies with SPR could only be performed in aqueous buffer solutions. A solution to this problem could be either the synthesis of more water-soluble ligands, or performing both imprinting and binding studies in ethanol (or other suitable organic solvent). The latter would, however, require a chemically more robust SPR setup, with a fluidics unit that can stand organic solvent well without giving excessive drift in the SPR response. However, it will be evident that the first alternative is more attractive for practical applications.

Our results point out also the role of the pyridyl moiety in the formation of imprinted layers with moderate affinity, either through hydrogen bonding and/or through the establishment of halogen bonds with the iodine atoms of $\mathbf{T 4}$. The relative importance of these two interactions could be investigated by using T4 analogues dehalogenated to different degrees, and will be the object of further studies.

\section{Acknowledgments}

Financial support from the Academy of Finland under the projects HaloSENSE (No. 141558) and BioHal (No. 260565) and from VTT, the Technical Research Centre of Finland, under the project HaloSENSE is gratefully acknowledged. The authors are also grateful to Heli Nygren (VTT Biotechnology) for performing the ESI-MS analyses of the pyridyl ligands.

\section{References and Notes}

1. Yen, P.M. Physiological and molecular basis of thyroid hormone action. Physiolog. Rev. 2001, 81, 1097-1142.

2. Schimmel, M.; Utiger, R.D. Thyroidal and peripheral production of thyroid hormones. Review of recent findings and their clinical implications. Ann. Intern. Med. 1977, 87, 760-768.

3. Sandler, B.; Webb, P.; Apriletti, J.W.; Huber, B.R.; Togashi, M.; Cunha, S.T.; Juric, S.; Nilsson, S.; Wagner, R.; Fletterick, R.J.; et al. Thyroxine-thyroid hormone receptor interactions. J. Biol. Chem. 2004, 279, 55801-55808. 
4. Wagner, R.L.; Apriletti, J.W.; McGrath, M.E.; West, B.L.; Baxter, J.D.; Fletterick, R.J. A structural role for hormone in the thyroid hormone receptor. Nature 1995, 378, 690-697.

5. Midgley, J.E.M. Direct and indirect free thyroxine assay methods: Theory and practice. Clin. Chem. 2010, 7, 1353-1363.

6. Zhou, A.; Wei, Z.; Read, R.J.; Carrell, R.W. Structural mechanism for the carriage and release of thyroxine in the blood. Proc. Natl. Acad. Sci. USA 2006, 103, 13321-13326.

7. Miyata, M.; Sato, T.; Kai, H. Role of the glutamic acid 54 residue in transthyretin stability and thyroxine binding. Biochemistry 2010, 49, 114-123.

8. Eneqvist, T.; Lundberg, E.; Karlsson, A.; Huang, S.; Santos, C.R.A.; Power, D.M.; Sauer-Eriksson, A.E. High resolution crystal structures of piscine transthyretin reveal different binding modes for triiodothyronine and thyroxine. J. Biol. Chem. 2004, 279, 26411-26416.

9. Metrangolo, P.; Meyer, F.; Pilati, T.; Resnati, G.; Terraneo, G. Halogen bonding in supramolecular chemistry. Angew. Chem. Int. Ed. 2008, 47, 6114-6127.

10. Crisp, T.M.; Clegg, E.D.; Cooper, R.L.; Wood, W.P.; Anderson, D.G.; Baetcke, K.P.; Hoffmann, J.L.; Morrow, M.S.; Rodier, D.J.; Schaeffer, J.E.; et al. Environmental endocrine disruption: An effects assessment and analysis. Environ. Health Perspect. 1998, 106, 11-56.

11. Wu, F.-B.; Han, S.-Q.; Xu, T.; He, Y.-F. Sensitive time-resolved fluoroimmunoassay for simultaneous detection of serum thyroid-stimulating hormone and total thyroxin with Eu and $\mathrm{Sm}$ as labels. Anal. Biochem. 2003, 314, 87-96.

12. Marchesini, G.R.; Meulenberg, E.; Haasnoot, W.; Mizuguchi, M.; Irth, H. Biosensor recognition of thyroid-disrupting chemicals using transport proteins. Anal. Chem. 2006, 78, 1107-1114.

13. Marchesini, G.R.; Koopal, K.; Meulenberg, E.; Haasnoot, W.; Irth, H. Spreeta-based biosensor assays for endocrine disruptors. Biosens. Bioelectron. 2007, 22, 1908-1915.

14. Mastichiadis, C.; Petrou, P.S.; Christofidis, I.; Misiakos, K.; Kakabakos, S.E. Bulk fluorescence light blockers to improve homogeneous detection in capillary-waveguide fluoroimmunosensors. Biosens. Bioelectron. 2009, 24, 2735-2739.

15. Shahgaldian, P.; Hegner, M.; Pieles, U. Cyclodextrin self-assembled monolayer (SAM) based surface plasmon resonance (SPR) sensor for enantioselective analysis of thyroxine. J. Incl. Phenom. Macrocycl. Chem. 2005, 53, 35-39.

16. Piletsky, S.; Turner, A. Molecular Imprinting of Polymers. Available online: http://www.landesbioscience.com/books/iu/id/864/?nocache=175958218 (accessed on 31 January 2013).

17. Vasapollo, G.; Del Sole, R.; Mergola, L.; Lazzoi, M.R.; Scardino, A.; Scorrano, S.; Mele; G. Molecularly imprinted polymers: Present and future prospective. Int. J. Mol. Sci. 2011, 12, 5908-5945.

18. Tappura, K.; Vikholm-Lundin, I.; Albers, W.M. Lipoate-based imprinted self-assembled molecular thin films for biosensor applications. Biosens. Bioelectron. 2007, 22, 912-919.

19. Corradi, E.; Meille, S.V.; Messina, M.T.; Metrangolo, P.; Resnati, G. Halogen bonding versus hydrogen bonding in driving self-assembly processes. Angew. Chem. Int. Ed. 2000, 39, 1782-1786.

20. Albers, W.M.; Vikholm-Lundin, I. Surface Plasmon Resonance on Nano-Scale Organic Films. In Nano-Bio-Sensing; Carrara, S., Ed.; Springer Verlag: New York, NY, USA, 2010. 
21. ACD/ChemSketch Reference Manual version 12.0. Available online: http://exordio.qfb.umich. mx/CHEMSK_R.pdf (accessed on 31 January 2013).

22. Packing of the ligands was assessed with the OPLS force field with standard Lennard-Jones potentials and electrostatic charges calculated by PM3 [23,24].

23. Kaminski, G.A.; Friesner, R.A.; Tirado-Rives, J.; Jorgensen, W.L. Evaluation and reparametrization of the OPLS-AA force field for proteins via comparison with accurate quantum chemical calculations on peptides. J. Phys. Chem. B 2001, 105, 6474-6487.

24. Stewart, J.J.P. Optimization of parameters for semi-empirical methods 1. Method. J. Comp. Chem. 1989, 10, 209-220.

25. Biacore 3000 Instrument Handbook. Available online: http://labs.idi.harvard.edu/springer/ uploads/Biacore3000InstrumentHandbookweb.pdf (accessed on 31 January 2013).

26. Sips, R. J. J. Combined form of Langmuir and Freundlich equations. Chem. Phys. 1948, 16, 490-495.

27. Liang, H.; Miranto, H.; Granqvist, N.; Sadowski, J.W.; Viitala, T.; Wang, B.; Yliperttula, M. Surface plasmon resonance instrument as a refractometer for liquids and ultrathin films. Sens. Act. B 2010, 149, 212-220.

(C) 2013 by the authors; licensee MDPI, Basel, Switzerland. This article is an open access article distributed under the terms and conditions of the Creative Commons Attribution license (http://creativecommons.org/licenses/by/3.0/). 